\title{
Polarimetric Doppler Spectrum of Backscattered Echoes From Nonlinear Sea Surface Damped by Natural Slicks
}

\author{
Pengju Yang, ${ }^{\mathrm{a}, *}$, Lixin $\mathrm{Guo}^{\mathrm{a}}$ \\ ${ }^{a}$ School of Physics and Optoelectronic Engineering, Xidian University, Xi'an 710071, \\ China.
}

\begin{abstract}
Based on the Lombardini et al. model that can predict the hydrodynamic damping of rough sea surfaces in the presence of monomolecular slicks and the "choppy wave" model (CWM) that can describe the nonlinear interactions between ocean waves, the modeling of time-varying nonlinear sea surfaces damped by natural or organic sea slicks is presented in this paper. The polarimetric scattering model of second-order small-slope approximation (SSA-II) with tapered wave incidence is utilized for evaluating co- and cross-polarized backscattered echoes from clean and contaminated CWM nonlinear sea surfaces. The influence of natural sea slicks on Doppler shift and spectral bandwidth of radar sea echoes is investigated in detail by comparing the polarimetric Doppler spectra of contaminated sea surfaces with those of clean sea surfaces. A narrowing of Doppler spectra in the presence of oil slicks is observed for both co- and crosspolarization, which is qualitatively consistent with wave-tank measurements. Simulation results also show that the Doppler shifts in slicks can increase or decrease, depending on incidence angles and polarizations.
\end{abstract}

Keywords: Doppler spectrum, electromagnetic scattering, natural sea slicks, sea surfaces, second-order small-slope approximation (SSA-II)

2010 MSC: 00-01, 99-00

${ }^{*}$ Corresponding author
Email address: pjyang@yeah.net (Pengju Yang)

Preprint submitted to Journal of ${ }^{A} T_{E} X$ Templates

July 17, 2016

(C) 2015. This manuscript version is made available under the Elsevier user license http://www.elsevier.com/open-access/userlicense/1.0/ 


\section{INTRODUCTION}

2 Doppler spectrum of radar echoes from rough sea surfaces is a promising 3 technique in many areas such as ocean wave spectra estimation [ $\mathbb{\square},[\vec{Z}]$, ocean 4 surface wind retrieval [3, 4], sea surface wave height retrieval [5, 6], sea surface 5 current measurement $[\bar{\nabla}, \mathbf{\nabla}]$, etc., which carries much more information than the 6 average scattering coefficient of rough sea surfaces [9, [0, П]]. Due to the neglect of nonlinear interactions between ocean waves, the horizontal component of orbital motions of Bragg scatterers [[I2] due to long waves is absent in linear sea surface models. As a consequence, the Doppler spectrum of linear sea surfaces at moderate to large incidence angles has nothing to do with physical reality. To overcome the drawbacks of linear sea surface models, several nonlinear hydrodynamic models such as the Creamer model [1.3] and its reduced version [14], the "choppy wave" model (CWM) [15], the West model [16], the nonlinear fractal model [I7], the Lagrange model with linked components [18] etc., have been proposed for better describing the hydrodynamic surface interactions of ocean waves. On the basis of these nonlinear hydrodynamic models, Doppler spectrum of radar echoes from one-dimensional [1.9, [20, 21, [22, [2.3, 24] and twodimensional sea surfaces [ [25, [26, [27, [28, 14] has been extensively investigated by utilizing rigorous numerical methods or analytical approximate models. In these studies, it is demonstrated that at low-grazing angles Doppler spectrum is significantly affected by nonlinear hydrodynamic modulation. More precisely, nonlinear sea surfaces show larger Doppler shift and spectral bandwidth compared with the corresponding linear counterparts at moderate to large incidence angles.

However, only clean sea surfaces are considered in most of the studies including the aforementioned ones on Doppler spectrum of radar sea echoes. In 27 ocean environment, natural or man-made sea slicks are encountered frequently. ${ }_{28}$ Remote sensing of oil slicks floating on sea surfaces has received considerable 29 attention, and most of the research efforts on this topic have been devoted to so analyzing and processing remote sensing data, particularly by synthetic aper- 
ture radar (SAR) [29, 30, 31, 32, 33]. As a basis for remote sensing of oil slicks floating on sea surfaces, electromagnetic scattering modeling of sea surface in the presence of oil slicks has also been carried out [34, 35, 36, 37]. In [36, 37], the influence of oil slicks' damping on the roughness spectrum of sea surfaces has been investigated to evaluate the electromagnetic scattering signatures of oil-covered sea surfaces, indicating that the small-scale capillary waves of sea surfaces are strongly damped by oil slicks and that oil slicks' damping effect on the angular distribution of scattered intensity is also pronounced.

Motivated by oil slicks' strong damping effect on the small-scale capillary waves of sea surfaces, our focus in this paper is on natural sea slicks' damping effect on Doppler shift and spectral bandwidth of backscattered echoes from time-evolving sea surfaces, which is potentially valuable for remote sensing of natural sea slicks floating on sea surfaces. Due to the desirable properties such as analytical tractability and numerical efficiency, the nonlinear hydrodynamic model of CWM is adopted in this paper for describing the nonlinear interactions between ocean waves. In comparison with the classical model such as Kirchhoff approximation, small perturbation method, two scale model, etc., the modern analytical approximate model of second-order small-slope approximation (SSA-II) [I] takes into account facets' tilts modulation and second-order Bragg scattering, and thus can reasonably predict the depolarized scattering from rough sea surface both in and outside the plane of incidence. Therefore, the polarimetric scattering model of SSA-II is utilized in the present study for evaluating the polarimetric Doppler spectrum of backscattered echoes from clean and contaminated nonlinear CWM sea surfaces.

The remainder of this paper is organized as follows. In Section 2, oil slicks' damping effect on sea surfaces roughness spectrum is examined briefly, and the modeling of nonlinear sea surfaces in the presence of natural sea slicks is presented. Section 3 presents the polarimetric scattering model of SSA-II with tapered wave incidence for evaluating polarimetric Doppler spectrum of clean and contaminated nonlinear CWM sea surfaces. The numerical results of Doppler spectra of backscattered echoes from clean and contaminated sea 
In the existing literature, only a few models that can take into account oil slicks and predict the hydrodynamic damping of rough sea surfaces in the presence of surface oil films are available [38, 39, 40]. In the present study, we put emphasis on the investigation of the influence of natural or organic sea slicks' damping effect on Doppler spectrum of backscattered echoes from sea surfaces. Hence, the Lombardini et al. model suitable for monomolecular slicks [40] is adopted in this paper. According to the model proposed by Lombardini et al., the roughness spectrum of contaminated sea surfaces $S_{\text {cont }}$ is related to that of clean sea surfaces $S_{\text {clean }}$ by the following ratio [40, 37]

$$
S_{\text {cont }}\left(k ; \phi, U_{10}, E_{0}, \omega_{D}\right)=\frac{S_{\text {clean }}\left(k ; \phi, U_{10}\right)}{y_{s}\left(k ; E_{0}, \omega_{D}\right)}
$$

where $y_{s}$ is the damping ratio. $k$ and $\phi$ in polar coordinates denote the ocean wave wavenumber and the wave propagation direction, respectively. $U_{10}$ is the wind speed at a height of $10 \mathrm{~m}$. For insoluble films and a fully covered sea, the damping ratio $y_{s}$ is expressed by

$$
y_{s}\left(f, E_{0}, \omega_{D}\right)=\frac{1-2 \tau+2 \tau^{2}-X+Y(X+\tau)}{1-2 \tau+2 \tau^{2}-2 X+2 X^{2}}
$$

where

$$
\tau=\left(\frac{\omega_{D}}{2 \omega}\right)^{\frac{1}{2}} \quad X=\frac{E_{0} k^{2}}{\rho\left(2 \nu \omega^{3}\right)^{0.5}} \quad Y=\frac{E_{0} k}{4 \rho \nu \omega}
$$

are dimensionless quantities and

$$
f=\frac{\omega}{2 \pi}=\frac{\left(\varsigma k^{3} / \rho+g_{0} k\right)^{1 / 2}}{2 \pi}
$$

65 is the dispersion law. In (四)-(四), $\rho$ is the water density, $g_{0}$ is the acceleration of ${ }_{66}$ gravity, $\nu=10^{-6} \mathrm{~m} / \mathrm{s}$ the kinematic viscosity, $\varsigma=74 \times 10^{-3} \mathrm{~N} / \mathrm{m}$ is the surface 67 tension. $E_{0}$ denotes the elasticity modulus and $\omega_{D}$ represents a characteristic ${ }_{68}$ angular frequency, which depend on surface film characteristics. It should be 
In the present study, the roughness spectrum of sea surface proposed by Elfouhaily et al. is utilized for describing clean sea surfaces. The Elfouhaily spectrum in polar coordinates can be expressed as [41]

$$
S_{\text {clean }}\left(k, \phi, U_{10}\right)=S_{\text {clean }}(k) \Phi(\phi)
$$

where $S_{\text {clean }}(k)$ denotes the isotropic parts of clean sea surface roughness spectrum and can be expressed as

$$
S_{\text {clean }}(k)=k^{-3}\left(B_{L}(k)+B_{H}(k)\right)
$$

where $B_{L}$ denotes the long-wave curvature spectrum and $B_{H}$ represents the short-wave curvature spectrum. The detailed expressions of $B_{L}$ and $B_{H}$ can be found in [4]]. In Eq. (国), $\Phi(\phi)$ represents the angular spreading function. In this paper, the angular spreading function is chosen as follows

$$
\Phi(\phi)=\frac{1}{2 \pi}\left(1+\Delta(k) \cos \left(2\left(\phi-\phi_{w}\right)\right)\right)
$$

where $\phi_{w}$ is the wind direction with respect to the radar line of sight and

$$
\Delta(k)=\tanh \left(\frac{\ln 2}{4}+4\left(\frac{c(k)}{c\left(k_{p}\right)}\right)^{2.5}+0.13\left(\frac{u_{f}}{c\left(k_{m}\right)}\right)\left(\frac{c\left(k_{m}\right)}{c(k)}\right)^{2.5}\right)
$$

73 where $c(k)=\sqrt{g\left(1+k^{2} / k_{m}^{2}\right) / k}, k_{m}=370 \mathrm{rad} / \mathrm{m}, c\left(k_{p}\right)=U_{10} / \Omega_{c}$ with $\Omega_{c}$ 74 being inverse wave age, $c\left(k_{m}\right)=\sqrt{2 g / k_{m}} \approx 0.23 \mathrm{~m} / \mathrm{s}$, and $u_{f}$ is the friction 75 velocity.

With the knowledge of the roughness spectrum of clean or contaminated sea surfaces, the root-mean-square (RMS) slope $\sigma_{s}$ and RMS height $\sigma$ can be derived as, respectively

$$
\sigma_{s}=\sqrt{\int_{0}^{+\infty} k^{3} d k \int_{0}^{2 \pi} d \phi S(k, \phi)}
$$




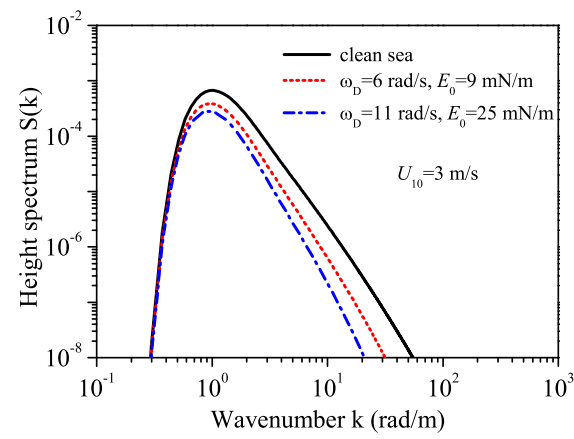

(a)

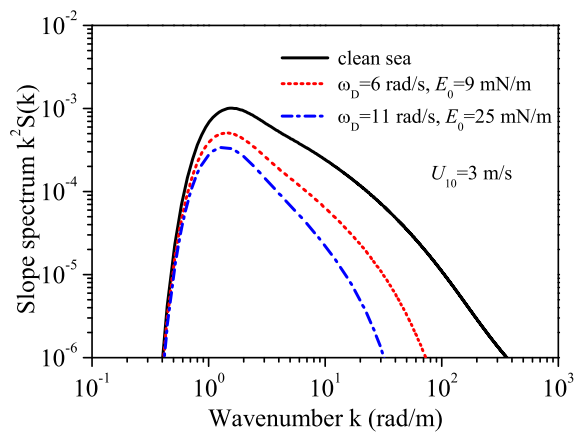

(b)

Figure 1: Height spectrum (isotropic part) and slope spectrum (isotropic part) of clean and contaminated sea surface versus wavenumber $k$. (a)Height spectrum, (b)slope spectrum.

$$
\sigma=\sqrt{\int_{0}^{+\infty} k d k \int_{0}^{2 \pi} d \phi S(k, \phi)}
$$

Figures $\mathrm{D(a)}$ and (b) present a comparison of the height spectrum (isotropic part) and slope spectrum (isotropic part) between clean and contaminated sea surfaces, respectively. In Fig. 四, $S(k)$ can be either $S_{\text {clean }}(k)$ or $S_{\text {cont }}(k)$. The wind fetch involved in Elfouhaily et al. spectrum is set as 300 km. In Fig. س, we consider two types of natural or organic sea surface films with parameters $\left\{\omega_{\mathrm{D}}=6 \mathrm{rad} / \mathrm{s}, E_{0}=9 \mathrm{mN} / \mathrm{m}\right\}$ and $\left\{\omega_{\mathrm{D}}=11 \mathrm{rad} / \mathrm{s}, E_{0}=25 \mathrm{mN} / \mathrm{m}\right\}$, respectively. It needs to be noted that these values were retrieved from experiments conducted in the Sicilian Channel and the Gulf of Maine [40]. From Fig. (1, we can observe that oil film strongly damps the high frequency components corresponding to the small-scale waves of sea surfaces. In addition, we can also find that oil film with parameter $\left\{\omega_{\mathrm{D}}=11 \mathrm{rad} / \mathrm{s}, E_{0}=25 \mathrm{mN} / \mathrm{m}\right\}$ has a stronger damping effect compared with oil film with parameter $\left\{\omega_{\mathrm{D}}=6 \mathrm{rad} / \mathrm{s}, E_{0}=9 \mathrm{mN} / \mathrm{m}\right\}$. Comparing Figures $\mathrm{D}(\mathrm{a})$ with (b), we can find that the influence of oil slicks on slope spectrum is much stronger in comparison with the height spectrum of sea surface.

Oil film's damping effect can exert nonnegligible influence on the geometrical 
In the present study, the contaminated and clean sea surfaces are generated with the roughness spectrum of contaminated sea surfaces $S_{\text {cont }}$ and that of clean sea surfaces $S_{\text {clean }}$, respectively. The spectral method under spatially homogeneous and time-stationary hypothesis is utilized for generating rough sea surface by taking into account its high efficiency, since the procedure of generating rough sea surface can be realized by fast Fourier transform. The generated rough sea surfaces including clean and contaminated sea, in essence, are realizations of Gaussian random process. All of the components of linear sea surfaces in Fourier domain are completely independent of random phases. This implies Gaussian statistics for sea surface elevations and their derivatives. The spatial Fourier amplitude at any time $t$ can be expressed as follows

$A(\bar{k}, t)=\gamma(\bar{k}) \sqrt{S(k, \phi) \delta k_{x} \delta k_{y}} \exp (i \omega t)+\gamma(-\bar{k})^{*} \sqrt{S(k, \pi-\phi) \delta k_{x} \delta k_{y}} \exp (-i \omega t)$

structure of sea surfaces such as surface heights and slopes, and on the statistical characteristics of sea surfaces such as surface RMS height and RMS slope. A detailed discussion of oil's damping effect on sea surface heights and slopes can be found in Pinel's work [37], and the main conclusions are listed here. Specifically, both surface heights and slopes of contaminated sea surfaces are smaller compared with clean sea surfaces. Moreover, the sea surface heights and slopes of contaminated sea surfaces with oil parameter $\left\{\omega_{\mathrm{D}}=11 \mathrm{rad} / \mathrm{s}, E_{0}=25 \mathrm{mN} / \mathrm{m}\right\}$ are smaller than those with $\left\{\omega_{\mathrm{D}}=6 \mathrm{rad} / \mathrm{s}, E_{0}=9 \mathrm{mN} / \mathrm{m}\right\}$. $A(\bar{k}, t)=\gamma(\bar{k}) \sqrt{S(k, \phi) \delta k_{x} \delta k_{y} \exp (i \omega t)+\gamma(-k)} \sqrt{S(k, \pi-\phi) \delta k_{x} \delta k_{y}} \exp (-i \omega t)$

where $\bar{k}=\left(k_{x}, k_{y}\right)=(k, \phi)$ is spatial wave vector. It should be noted that all the over bar symbol below in this paper represent vector symbol. $S(k, \phi)$ is sea surface roughness spectrum. $\gamma(\bar{k})$ is a complex Gaussian random series with zero mean value and unit variance. The sampling interval along $x$ direction is $\delta k_{x}=2 \pi / L_{x}$ which is determined by Nyquist sampling criterion with $L_{x}$ being the length of sea surface along $x$ direction. Similarly, the sampling interval along $y$ direction $\delta k_{y}=2 \pi / L_{y}$ with $L_{y}$ being the length of sea surface along $y$ direction. According to the gravity-capillary dispersion relation, the angular frequency $\omega$ is related to the spatial wavenumber $k=|\bar{k}|$ by 
$\omega=\sqrt{g_{0} k\left(1+k^{2} / k_{m}^{2}\right)}$ with $g_{0}$ being the gravity acceleration constant.

Then, the linear sea surface elevation $h(\bar{r}, t)$ at spatial potion $\bar{r}$ for time $t$ can be expressed by

$$
h(\bar{r}, t)=\sum_{\bar{k}} A(\bar{k}, t) \exp (i \bar{k} \cdot \bar{r})
$$

Eq.(ए2) can be efficiently accomplished by inverse fast Fourier transform. To ensure that $h(\bar{r}, t)$ is real, $A(\bar{k})$ is required to satisfy the conjugate symmetry about the origin as follows

$$
A\left(k_{x}, k_{y}\right)=A^{*}\left(-k_{x},-k_{y}\right), A\left(k_{x},-k_{y}\right)=A^{*}\left(-k_{x}, k_{y}\right)
$$

The nonlinear hydrodynamic model of CWM is adopted in this paper for describing nonlinear interactions between wave components, which is analytically tractable and numerically efficient. The CWM is based on a Lagrangian description of ocean waves and can be constituted by horizontal displacement of Hilbert transform of a reference linear surface. Given a linear sea surface $h(\bar{r}, t)$ and its Hilbert transform, the CWM nonlinear sea surface $\tilde{h}(\bar{r}, t)$ is defined by [1.5]

$$
\tilde{h}(\bar{r}+\bar{D}(\bar{r}, t), t)=h(\bar{r}, t)
$$

The displacement can be written as [15]

$$
\bar{D}(\bar{r}, t)=\int-i \frac{\bar{k}}{k} \hat{h}(\bar{k}, t) \exp (i \bar{k} \cdot \bar{r}) d \bar{k}
$$

where

$$
\hat{h}(\bar{k}, t)=\frac{1}{(2 \pi)^{2}} \int d \bar{r} \exp (-i \bar{k} \cdot \bar{r}) h(\bar{r}, t)
$$

is the spatial Fourier transform. The model of CWM is numerically efficient as it can be entirely generated by fast Fourier transform.

\section{POLARIMETRIC SCATTERING MODEL OF SSA-II MODEL FOR CALCULATING DOPPLER SPECTRUM}

The small-slope approximation theory consists of a basic approximation of the theory (SSA-I) and second-order corrections to it (SSA-II) and represents a 


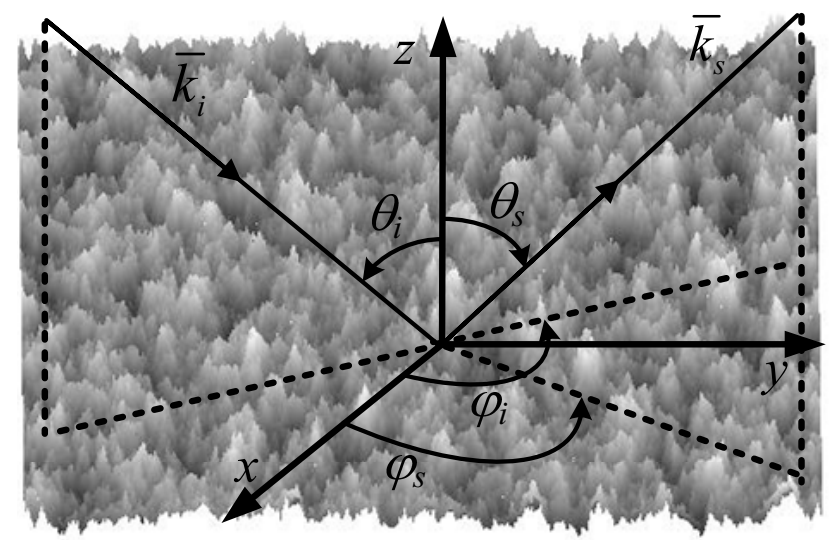

Figure 2: Geometry of sea surface scattering problem.

systematic expansion of a scattering amplitude with respect to slopes of rough surface, which has been successfully applied to evaluate microwave scattering from rough sea surfaces. The modern analytical approximate model of SSA-II takes into mutual transformation of the two linear polarization states caused by facets tilts as well as the second-order Bragg scattering, and thus can predict the depolarized scattering from rough sea surface both in and outside the plane of incidence [III].

To the authors' knowledge, two different ways can be found in literature for dealing with electromagnetic scattering from rough sea surfaces covered by oil films. The first way just considers the damping effect of oil film on sea surface but ignore the thickness of oil film, as done in [35] by Nunziata et al. For this approach, oil film's damping effect is taken into account by modifying sea surface roughness spectrum with the use of Marangoni damping theory suitable for describing monomolecular organic film floating on ocean surface whose thickness is extremely small compared to electromagnetic wavelength. It should be noted that Nunziata's way implies an assumption that sea biogenic slicks have a zero-thickness. Thus, Nunziata's way cannot take into account multiple scattering between air-oil and oil-water interface. In principle, this 
assumption depends on incidence angles, since multiple scattering between airoil and oil-water interface becomes pronounced under large incidence angles.

The second one considers both oil film's damping effect and oil film's thickness, as done in [37] by Pinel et al. In fact, Pinel's way [37] significantly differs from Nunziata'way [3.5]. More specifically, the former is a two-layered rough surface scattering problem, whereas the latter is a single-layered rough surface scattering problem. One obvious advantage of Pinel's approach [37] is that it can take into account multiple scattering between air-oil and oil-water interface. In addition, the absorption of incident electromagnetic energy due to oil film can also been considered by Pinel's approach. However, the rigorous numerical methods such as method of moments (MoM) and its improved versions encounter convergence problems when dealing with electromagnetic scattering problems of sea surfaces covered by monomolecular organic film, since the thickness of monomolecular organic film is extremely small compared to electromagnetic wavelength.

In the present study, monomolecular organic film is considered with emphasis on its influence on Doppler spectrum of backscattered echoes from 2-D timevarying ocean surfaces. Thus, we adopt Nunziata's [35] way by modifying sea surface roughness spectrum with the use of Marangoni damping theory to take into account oil films damping effect on ocean waves, in which oil film's damping effect on ocean waves is considered but the thickness of oil film is ignored. This implies that the effects of oil slicks on sea surface geometrical structures such as surface heights and slopes can be taken into account with the damping ratio proposed by Lombardini et al. [40]. Therefore, the polarimetric scattering model of SSA-II can be utilized for calculating co-polarized (HH, VV) and crosspolarized $(\mathrm{HV}, \mathrm{VH})$ radar echoes from both clean and contaminated sea surfaces.

The geometry of the electromagnetic wave scattering problem is illustrated in Fig. 叉. The configuration angles $\left(\theta_{i}, \varphi_{i}, \theta_{s}, \varphi_{s}\right)$ represent the incident angle, incident azimuth angle, scattering angle, and scattering azimuth angle, respectively. $\bar{k}_{i}=\left(\bar{k}_{0}, q_{0}\right)$ and $\bar{k}_{s}=(\bar{k}, q)$ are the incident wave vector and scattering wave vector, respectively. In this paper, a tapered plane wave in cho- 
sen as the incident field to reduce the edge effect caused by the limited surface size of $L_{x} \times L_{y}$, which is expressed as [42]

$$
\begin{gathered}
\bar{E}_{i}(\bar{r})=T(\bar{r}) \exp \left(i \bar{k}_{i} \cdot \bar{r}\right) \\
T(\bar{r})=\exp \left[i\left(\bar{k}_{i} \cdot \bar{r}\right) w\right] \exp \left(-t_{x}-t_{y}\right)
\end{gathered}
$$

where

$$
\begin{gathered}
t_{x}=\frac{\left(x \cos \theta_{i} \cos \varphi_{i}+y \cos \theta_{i} \sin \phi_{i}+z \sin \theta_{i}\right)^{2}}{g^{2} \cos ^{2} \theta_{i}} \\
t_{y}=\frac{\left(-x \sin \varphi_{i}+y \cos \varphi_{i}\right)^{2}}{g^{2}} \\
w=\frac{1}{k_{i}{ }^{2}}\left(\frac{2 t_{x}-1}{g^{2} \cos ^{2} \theta_{i}}+\frac{2 t_{y}-1}{g^{2}}\right)
\end{gathered}
$$

and $g$ is the parameter that controls the tapering of the incident wave. Thus, the original scattering amplitude of the SSA-II model can be expressed as follows after introducing the tapered incident wave

$$
\begin{aligned}
S\left(\bar{k}, \bar{k}_{0}\right)= & \frac{2 \sqrt{q_{0} q}}{\left(q_{0}+q\right) \sqrt{P_{\text {inc }}}} \int \frac{d \bar{r}}{(2 \pi)^{2}} T(\bar{r}) \times \exp \left(-i\left(\bar{k}-\bar{k}_{0}\right) \cdot \bar{r}+i\left(q+q_{0}\right) h(\bar{r})\right) \\
& \times\left(B\left(\bar{k}, \bar{k}_{0}\right)-\frac{i}{4} \int M\left(\bar{k}, \bar{k}_{0} ; \bar{\xi}\right) h(\bar{\xi}) \times \exp (i \bar{\xi} \cdot \bar{r}) d \bar{\xi}\right)
\end{aligned}
$$

where

$$
h(\bar{\xi})=\frac{1}{(2 \pi)^{2}} \int h(\bar{r}) \exp (-i \bar{\xi} \cdot \bar{r}) d \bar{r}
$$

is the Fourier transform of the surface elevation. $P_{i n c}$ is the incident wave power captured by sea surface. The kernel functions of $B$ and $M$ are $2 \times 2$ matrices describing mutual transformations of the EM waves of different polarizations, which depend primarily on the polarizations, configuration angles, and the permittivity of the lower medium. It can be proved that, in a general case, $M\left(\bar{k}, \bar{k}_{0} ; 0\right)=0$. For this reason, the term related to the function $M$ in Eq. (E2) is, in fact, proportional to the slopes of rough surface rather than to the elevations themselves. This term provides a correction to the first-order small 


(1)

slope approximation (SSA-I). Thus, Eq. (ए2) corresponds to the SSA-II, and using $M=0$ in it results in the SSA-I. A detailed derivation and the corresponding kernel functions in the SSA framework can be found in [4.3].

Different from linear sea surfaces model whose spatial sample intervals are uniform, the spatial sample intervals of the CWM nonlinear sea surfaces model are not uniform. Therefore, Eq.([2Z) cannot be directly applied to CWM nonlinear sea surfaces. When the SSA-II model is applied to the CWM nonlinear sea surfaces model, the integral variables $\bar{r}$ in Eq.([2Z) should be replaced by $\tilde{r}=\bar{r}+\bar{D}(\bar{r})$. The Jacobian transformation of $J$ from $\bar{r}$ to $\tilde{r}$ can be utilized to accomplish this change of integral variables. Therefore, to apply the SSA-II model to CWM nonlinear sea surfaces, Eq.(피) should be rewritten as

$$
\begin{aligned}
S\left(\bar{k}, \bar{k}_{0}\right)= & \frac{2 \sqrt{q_{0} q}}{\left(q_{0}+q\right) \sqrt{P_{\text {inc }}}} \int \frac{d \bar{r}}{(2 \pi)^{2}}|J(\bar{r}, t)| \times T(\bar{r}+\bar{D}(\bar{r}, t)) \\
& \quad \exp \left(-i\left(\bar{k}-\bar{k}_{0}\right) \cdot(\bar{r}+\bar{D}(\bar{r}, t))+i\left(q+q_{0}\right) h(\bar{r})\right) \\
& \quad \times\left(B\left(\bar{k}, \bar{k}_{0}\right)-\frac{i}{4} \int M\left(\bar{k}, \bar{k}_{0} ; \bar{\xi}\right) h(\bar{\xi}) \times \exp (i \bar{\xi} \cdot \bar{r}) d \bar{\xi}\right)
\end{aligned}
$$

where

$$
J(\bar{r}, t)=\left|\begin{array}{cc}
1+\partial D_{x}(\bar{r}, t) / \partial x & \partial D_{x}(\bar{r}, t) / \partial y \\
\partial D_{y}(\bar{r}, t) / \partial x & 1+\partial D_{y}(\bar{r}, t) / \partial y
\end{array}\right|
$$

The Doppler spectrum is defined as the power spectral density of the random time-varying scattering amplitude and can be evaluated by utilizing a standard spectral estimation technique by the following expression[2:3]

$$
S(f)=\left\langle\frac{1}{T}\left|\int_{0}^{T} S\left(\bar{k}, \bar{k}_{0} ; t\right) e^{-i 2 \pi f t} d t\right|^{2}\right\rangle
$$

where $S\left(\bar{k}, \bar{k}_{0} ; t\right)$ is the scattering amplitude from sea surfaces at time $t$. The angular bracket denotes the ensemble average over random surface realizations and $T$ represents the sea surface evolution time. In what follows, we consider only the backscattering case, i.e. $\theta_{s}=\theta_{i}, \varphi_{i}=0^{\circ}$, and $\varphi_{s}=180^{\circ}$.

The Doppler shift $f_{c}$, i.e. the spectral centroid, and the bandwidth of Doppler spectrum $f_{w}$ are defined by the following expressions to quantitatively 
measure Doppler spectrum

$$
\begin{gathered}
f_{c}=\frac{\int f S(f) d f}{\int S(f) d f} \\
f_{w}=\sqrt{\frac{\int\left(f-f_{c}\right)^{2} S(f) d f}{\int S(f) d f}}
\end{gathered}
$$

The Doppler shift $f_{c}$, which is an important parameter of Doppler spectrum, corresponds to a power-weighted mean line-of-sight velocity of the scatterers. The bandwidth of Doppler spectrum $f_{w}$ is determined by the variance of the velocity distribution of the scattering facets at sea surfaces.

\section{NUMERICAL RESULTS AND DISCUSSION}

In the following simulations, the influence of natural sea slicks with parameter $\left\{\omega_{\mathrm{D}}=11 \mathrm{rad} / \mathrm{s}, E_{0}=25 \mathrm{mN} / \mathrm{m}\right\}$ on Doppler spectrum of sea echoes is investigated in detail. The incident wave frequency is $1.2 \mathrm{GHz}$. At the frequency of $1.2 \mathrm{GHz}$, the relative permittivity of sea surface is $\varepsilon_{r}=73.2+i 67.2$ at sea water temperature of $20^{\circ} \mathrm{C}$ and salinity of 30 parts per thousand in terms of Debye expression [44]. The sampling interval is $\lambda / 8$ where $\lambda$ is electromagnetic wavelength. The sizes of sea surfaces are $L_{x}=L_{y}=128 \lambda=32 \mathrm{~m}$ and $L_{x}=L_{y}=256 \lambda=64 \mathrm{~m}$ for wind speed $U_{10}=3 \mathrm{~m} / \mathrm{s}$ and $U_{10}=5 \mathrm{~m} / \mathrm{s}$, respectively. The tapering parameter $g$ is set to $L_{x} / 6$. The wind fetch is taken as $300 \mathrm{~km}$. The time step is set to $0.02 \mathrm{~s}$ to obtain sufficient unambiguous Doppler bandwidth, and each realization of Doppler spectrum is performed on 256 time samples to obtain sufficient Doppler spectral resolution. The final Doppler spectrum is an ensemble average over 50 realizations of sea surfaces.

Figures $\mathbf{3}$ and $\mathbf{Q}$ present the average Doppler spectra of backscattered echoes from clean and contaminated CWM nonlinear sea surfaces with wind speed $U_{10}=3 \mathrm{~m} / \mathrm{s}$ and $U_{10}=5 \mathrm{~m} / \mathrm{s}$, respectively. For all simulations in Figures 3 and $\mathbf{\theta}$, the radar is looking upwind. For reference, the Bragg lines are also plotted with vertical short dotted lines, which are predicted by small perturbation method (SPM) at first order and can be calculated by $f_{B}= \pm \sqrt{g_{0} K_{B}} / 2 \pi$ where 

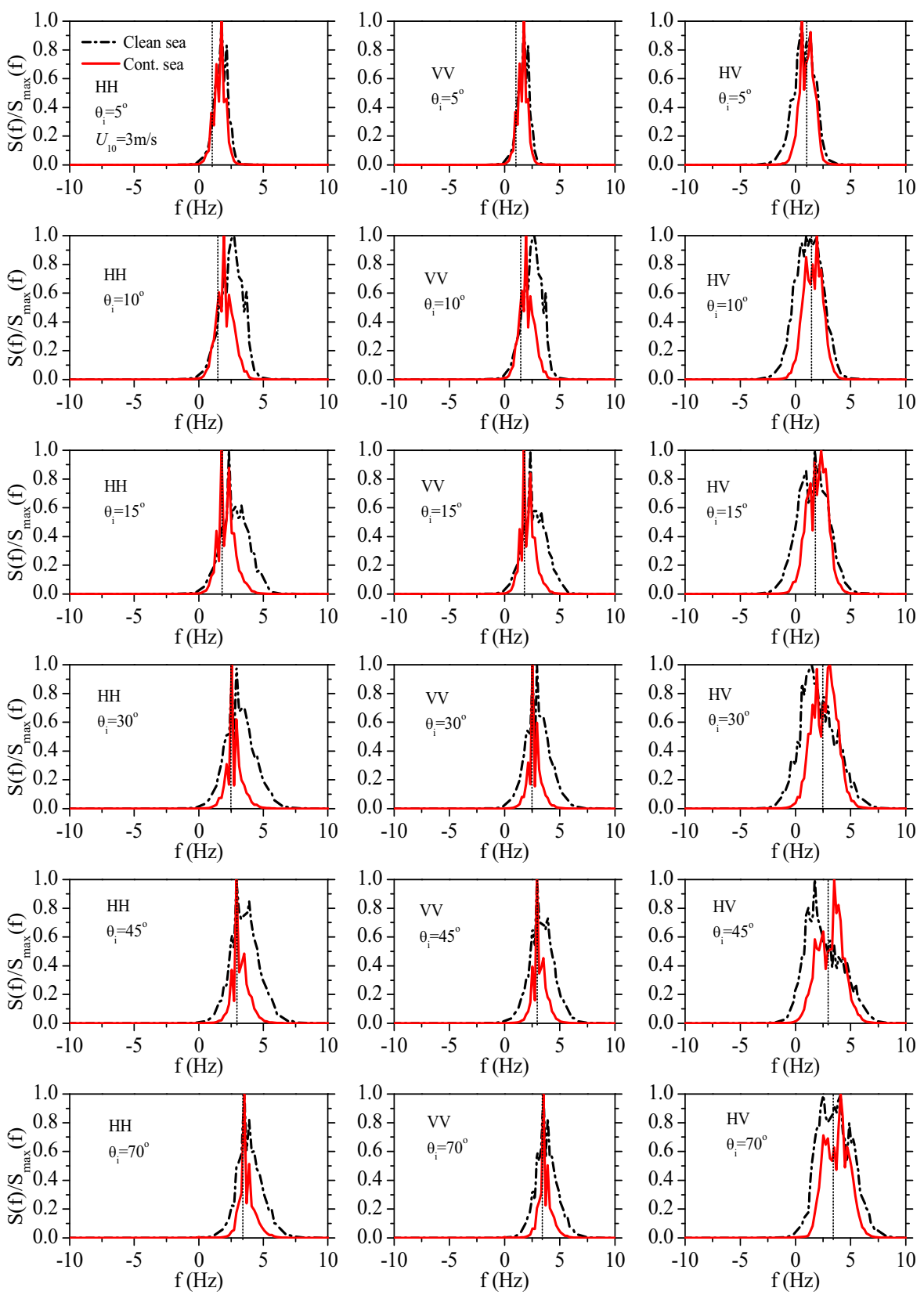

Figure 3: Average Doppler spectra of backscattered echoes from clean and contaminated CWM nonlinear sea surfaces with wind speed $U_{10}=3 \mathrm{~m} / \mathrm{s}$ at various incidence angles. The left column, middle column, and right column is for $\mathrm{HH}, \mathrm{VV}$, and HV polarization, respectively. The vertical short dotted lines represent Bragg lines. The radar is looking upwind. 

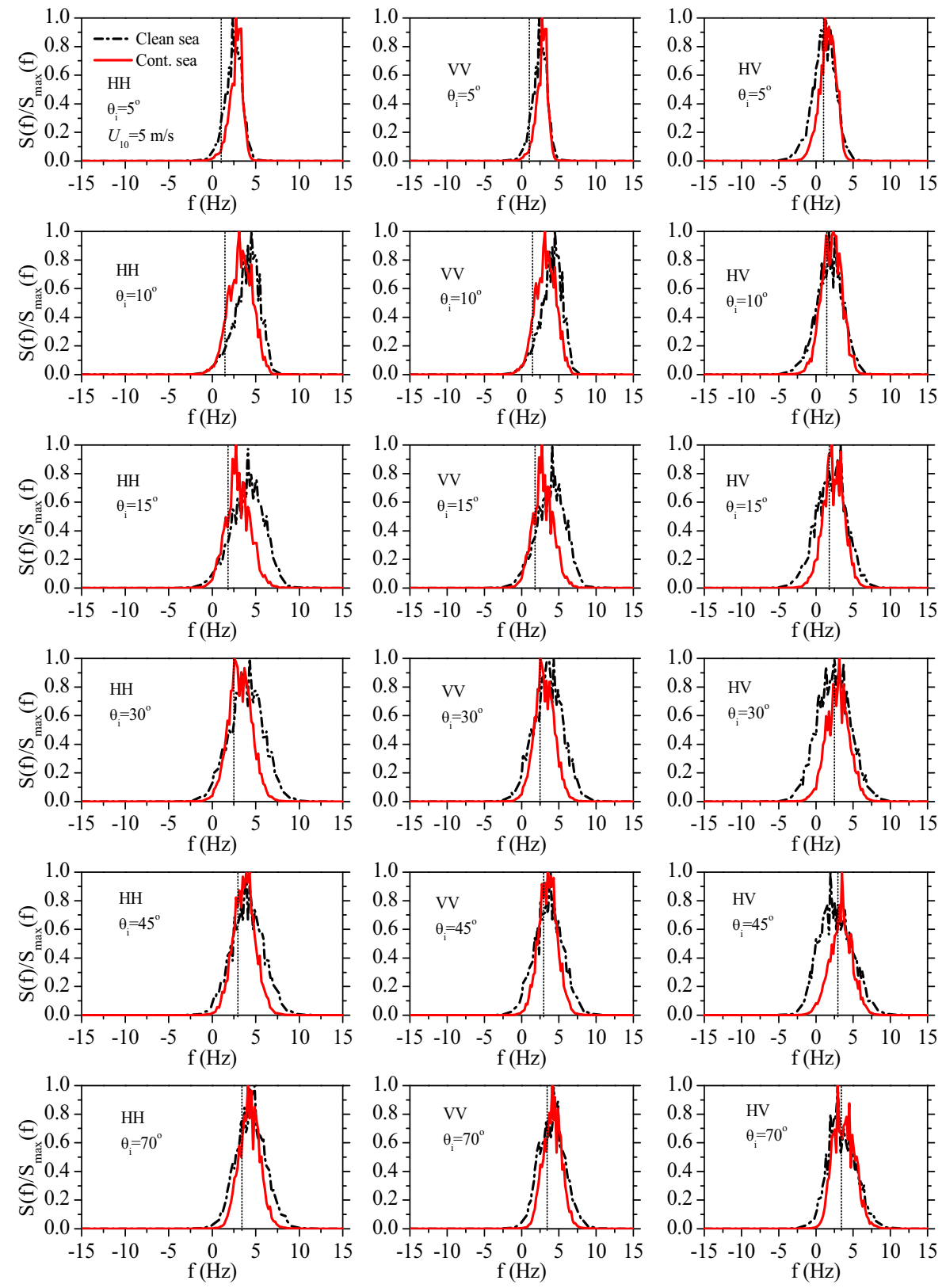

Figure 4: Similar to Fig. 而, but with wind speed $U_{10}=5 \mathrm{~m} / \mathrm{s}$. 
$K_{B}\left(\theta_{i}\right)=2 k_{0} \sin \left(\theta_{i}\right)$ is the Bragg wavenumber. The polarimetric scattering model of SSA-II is utilized for evaluating co-polarized (HH, VV) and crosspolarized ( $\mathrm{HV}, \mathrm{VH})$ scattering field from 2-D time-varying sea surfaces, since there is no difference of normalized Doppler spectrum predicted by SSA-I between $\mathrm{HH}$ and VV polarization. Due to the reciprocity in backscattering which means that $\mathrm{HV}$-polarized backscattering is equal to $\mathrm{VH}$-polarized backscattering, thus for cross-polarization only HV-polarized Doppler spectra are presented. In addition, rigorous numerical simulations have demonstrated that the Doppler spectra of linear sea surfaces are extremely narrow at low-grazing angles [2:3]. This is exceedingly unrealistic and is not consistent with measurements, because the linear sea surface model is just a collection of harmonics each propagating independently of others, in which the hydrodynamic interactions between ocean waves are ignored. Hence, it is necessary to adopt nonlinear sea surface model to investigate oil film's damping effect on Doppler spectra of radar sea echoes, in particular for Doppler spectra at moderate to large incidence angles. The linear sea surface model completely misses the horizontal components of orbital motions that shorter waves experience due to long waves. However, the CWM nonlinear sea surface does capture these orbital motions correctly by adding a horizontal displacement of Hilbert transform of a reference linear surface. Accordingly, the Doppler spectra at moderate to large incidence angles predicted by CWM nonlinear sea surfaces are much more consistent with radar measurements in comparison with linear sea surface model. Thus, the SSA-II model combined with the CWM nonlinear sea surface model is utilized in the present study to examine oil film's damping effect on Doppler spectrum signatures of radar sea echoes such as Doppler shift and Doppler spectral bandwidth.

Comparing the Doppler spectra of clean and contaminated sea surfaces illustrated in Figures $\mathbf{3}$ and $\mathbf{Q}$, we can observe that for both co-polarization and cross-polarization the Doppler spectra of contaminated sea surfaces are narrower than those of clean sea surfaces. That is, a narrowing of Doppler spectrum in slicks is observed regardless of incidence angles. To quantitatively compare the Doppler spectrum width between clean and contaminated sea surfaces, the 


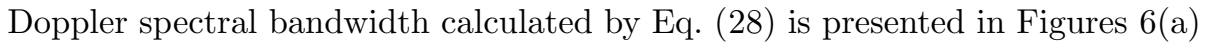
and (b)for wind speed $U_{10}=3 \mathrm{~m} / \mathrm{s}$ and $U_{10}=5 \mathrm{~m} / \mathrm{s}$, respectively. Obviously, the Doppler spectral bandwidths of contaminated sea surfaces are smaller than those of clean sea surfaces as depicted in Figures $6(\mathrm{a})$ and (b). This is due to the fact that Doppler spectral bandwidth depends mainly on the variance of orbital velocities of ocean waves. The orbital velocities of ocean waves are reduced by oil slicks, since the magnitude of the sea surface roughness spectrum is decreased due to oil slicks' damping effect. In addition, for contaminated sea surfaces, it is indicated that the difference of Doppler spectral bandwidth between $\mathrm{HH}$ and VV polarizations is almost indistinguishable. For both clean and contaminated sea surfaces, the Doppler spectral bandwidths for co-polarization are smaller for than those for cross-polarization.

In wind-wave tank measurements [45], the influence of natural sea slicks on Doppler spectrum of radar sea echoes has been investigated. During the experiments, measurements of radar backscattering were performed at X- and Kabands with fixed incidence angles $35^{\circ}$ and $53^{\circ}$, respectively. The scatterometers were operating at only VV polarization. Monomolecular slicks were produced by disturbing oleyl alcohol on water surface to simulate natural sea slicks. The wind-wave tank measurements have confirmed that, the Doppler spectrum in slicks is narrower in comparison with clean sea surfaces [4.5]. It should be noted that due to the severe computational burden our numerical simulations are limited to lower frequency band, i.e., L-band $(1.2 \mathrm{GHz})$, while the wind-wave tank measurements [45] were performed at much higher frequency bands, i.e., X- and Ka-bands. Thus, we cannot fairly compare our simulation results with the measurements [45]. Nonetheless, the simulation results are qualitatively consistent with the wave-tank measurements [45]. In addition, the effect of sea slicks on Doppler spectrum width at incidence angles of $0^{\circ}$ and $10^{\circ}$ was investigated by using an empirical formula of Doppler spectral bandwidth [46], indicating also that at low to moderate wind speeds the Doppler spectrum width in slicks is decreased due to the presence of oil slicks.

The Doppler spectral bandwidths of radar sea echoes are reduced due to the 
presence of sea slicks regardless of incidence angles, as indicated in the previous discussions. However, the variation of Doppler shift in the presence of oil slicks is very complicated in comparison with Doppler spectral bandwidth. More specifically, the simulation results indicate that the Doppler shift of radar seaechoes in the presence of natural sea slicks can decrease or increase, depending on incidence angle and polarization state. To our knowledge, for cross-polarization case, the investigation of oil slicks' damping effect on Doppler spectrum has not been reported in literature. From Figures 3 and $\mathbf{G}$, it is readily observed that the movement tendency of Doppler spectra peaks in slicks is different between co-polarization and cross-polarization. Moreover, for cross-polarization case at small to moderate incidence angles, the Doppler shifts of contaminated sea surfaces are obviously increased compared with those of clean sea surfaces.In what follows, we will discuss the variation of Doppler shift in the presence of oil slicks in detail.

For co-polarization case, it is indicated in Fig. 田 that for near-nadir configuration $\left(\theta_{i}=5^{\circ}\right)$ the Doppler spectra of contaminated sea surfaces at wind speed $U_{10}=5 \mathrm{~m} / \mathrm{s}$ are shifted to higher frequencies compared with those of the corresponding clean sea surfaces. For the lower wind speed $U_{10}=3 \mathrm{~m} / \mathrm{s}$, however, the Doppler spectra peaks of clean and contaminated sea surfaces can hardly be distinguished at incidence angle $\theta_{i}=5^{\circ}$. As incidence angle increases, the co-polarized Doppler spectra peaks in slicks are shifted to lower frequencies relative to those of clean sea surfaces as illustrated in Figures 3 and $⿴$ tor wind speed $U_{10}=3 \mathrm{~m} / \mathrm{s}$ and $U_{10}=5 \mathrm{~m} / \mathrm{s}$, respectively. To better measure the difference of Doppler shift between clean and contaminated sea surfaces, the Doppler shifts defined by Eq. ([28) are presented in Figures $\mathbf{Z}(\mathrm{a})$ and (b) for wind speed $U_{10}=3 \mathrm{~m} / \mathrm{s}$ and $U_{10}=5 \mathrm{~m} / \mathrm{s}$, respectively. From Fig. $\square(\mathrm{b})$, we can observe that at incidence angle $\theta_{i}=5^{\circ}$ the co-polarized Doppler shifts of contaminated sea surfaces are larger than those of clean sea surfaces with wind speed $U_{10}=5 \mathrm{~m} / \mathrm{s}$. However, the Doppler shifts of clean and contaminated sea surfaces are close to each other at incidence angle $\theta_{i}=5^{\circ}$ for the lower wind speed $U_{10}=3 \mathrm{~m} / \mathrm{s}$ as depicted in Fig. $\square(\mathrm{a})$. As incidence angle increases pro- 
gressively, the co-polarized Doppler shifts in slicks are decreased in comparison with clean sea surfaces. In particular at small to moderate incidence angles, the co-polarized Doppler shifts are significantly reduced due to the presence of sea slicks as illustrated in Figures $\square(\mathrm{a})$ and (b) for wind speed $U_{10}=3 \mathrm{~m} / \mathrm{s}$ and $U_{10}=5 \mathrm{~m} / \mathrm{s}$, respectively.

For cross-polarization case, the Doppler spectra peaks of contaminated sea surfaces are shifted to higher frequencies as depicted in Figures 3 and $\$$ for wind speed $U_{10}=3 \mathrm{~m} / \mathrm{s}$ and $U_{10}=5 \mathrm{~m} / \mathrm{s}$, respectively. As expected, the cross-polarized Doppler shifts of contaminated sea surfaces are larger than those of clean sea surfaces as illustrated in Figures $\mathbf{\square ( a )}$ and (b) for wind speed $U_{10}=3 \mathrm{~m} / \mathrm{s}$ and $U_{10}=5 \mathrm{~m} / \mathrm{s}$, respectively. Comparing Doppler shifts in slicks between co- and cross-polarizations, we can observe that the Doppler shifts in slicks can decrease or increase, which depends strongly on polarization. In addition, the difference of Doppler shift in slicks between co- and cross-polarization is much smaller in comparison with clean sea surfaces as illustrated in Figures प(a) and (b) for wind speed $U_{10}=3 \mathrm{~m} / \mathrm{s}$ and $U_{10}=5 \mathrm{~m} / \mathrm{s}$, respectively.

In the open ocean experiments carried out in 1997-1998 on the Black Sea [47, 48], it was indicated that the variations of Doppler shifts in slicks is very complicated. The VV-polarized radar measurements performed at X- and Kabands with fixed incidence angle $55^{\circ}$ showed that Doppler shifts in slicks can increase or decrease, depending on radar wavelength and surface film characteristics [47, 48]. It should be noted that our simulations are limited to L-band (1.2 $\mathrm{GHz}$ ) due to the severe computational burden encountered in large-scale Monte Carlo simulations, while the open ocean experiments were performed at X-and Ka-bands [47, 48]. Hence, our simulation results cannot be fairly compared with the measurements [47, 48]. In addition, at wind speed 7-9 m/s, a strong reduction of Doppler shifts in slicks at X-band is observed in the wind-wave tank measurements performed at incidence angle of $35^{\circ}$ for $\mathrm{VV}$ polarization [4.5].

Figure 5 show the comparison of normalized Doppler spectra of backscattered echoes from clean and contaminated CWM nonlinear sea surface. The wind speed is $U_{10}=3 \mathrm{~m} / \mathrm{s}$, and the radar is looking crosswind. From Figure 5 , 

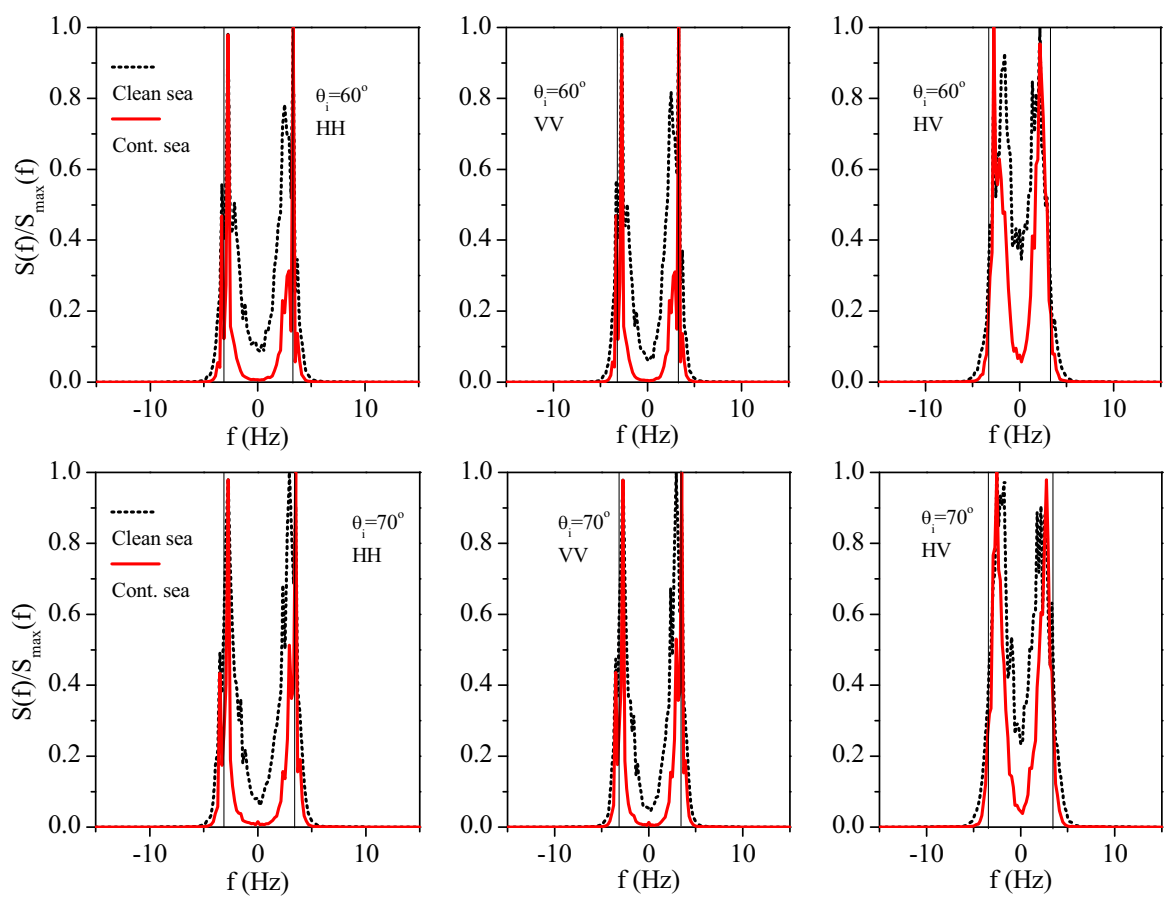

Figure 5: Comparison of normalized Doppler spectra of backscattered echoes from clean and contaminated CWM nonlinear sea surface at wind speed $U_{10}=3 \mathrm{~m} / \mathrm{s}$. The vertical solid lines represent Bragg lines. The radar is looking crosswind.

two obvious peaks can be observed for both co- and cross-polarization. For copolarization, the two peaks corresponding to the positive and negative frequencies are centered at first-order Bragg peaks produced by ocean waves moving toward and away from the radar. However, the two peaks for cross-polarization are not so much closer to the first-order Bragg peaks in comparison with copolarization case. Comparing the Doppler spectra of clean and contaminated sea surfaces, we can readily observe that the Doppler spectra of contaminated sea surfaces are narrower than those of clean sea surfaces. This can be attributed to the fact that Doppler spectral bandwidth depends mainly on the variance of orbital velocities of ocean waves, and that the orbital velocities of ocean waves are reduced due to the presence of sea slicks. In addition, the Doppler spectral 


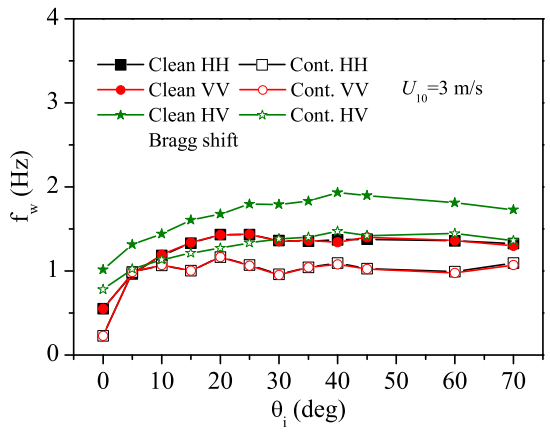

(a)

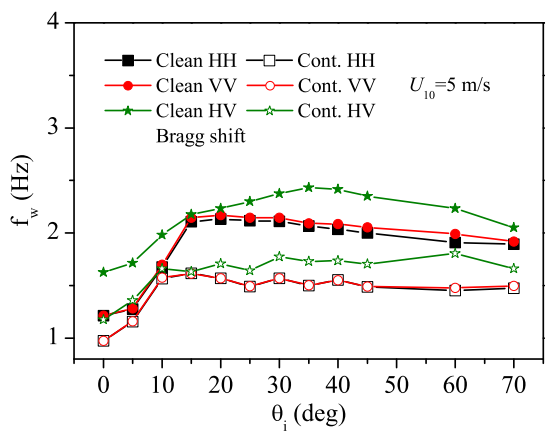

(b)

Figure 6: Comparison of Doppler spectral bandwidths for clean and contaminated sea surfaces. (a) $U_{10}=3 \mathrm{~m} / \mathrm{s}$. (b) $U_{10}=5 \mathrm{~m} / \mathrm{s}$.

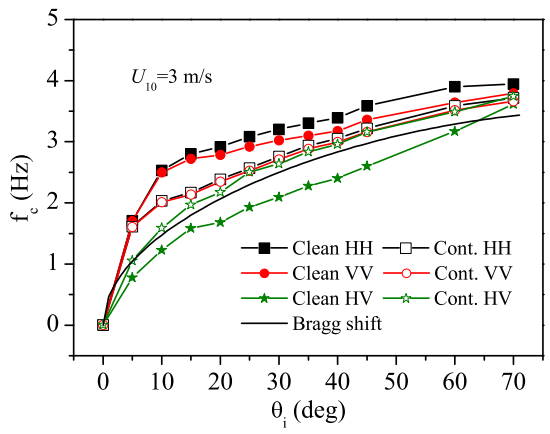

(a)

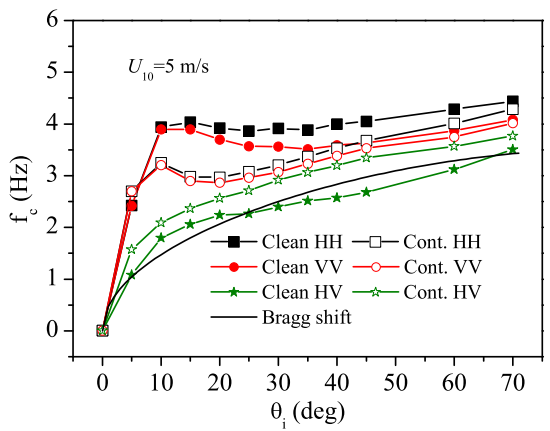

(b)

Figure 7: Comparison of Doppler shifts for clean and contaminated sea surfaces. (a) $U_{10}=$ $3 \mathrm{~m} / \mathrm{s}$. (b) $U_{10}=5 \mathrm{~m} / \mathrm{s}$.

\section{CONCLUSION}

In this paper, natural or organic sea slicks' damping effect on sea surface roughness spectrum is examined briefly, indicating that the heights and slopes 
of sea surfaces are reduced due to oil slicks' damping effect. On this basis, the influence of natural sea slicks on polarimetic Doppler spectra is investigated in detail by comparing the Doppler spectra of contaminated sea surfaces with those of clean sea surfaces. The polarimetric scattering model of SSA-II combined with CWM nonlinear sea surfaces model is utilized for evaluating the co-polarized and cross-polarized Doppler spectra of backscattered echoes from clean and contaminated CWM nonlinear sea surfaces.

A narrowing of Doppler spectra in the presence of oil slicks is observed for both co-polarization and cross-polarization, which is qualitatively consistent with wave-tank measurements performed at X- and Ka-bands [45]. However, the variation of Doppler shift in slicks is very complicated in comparison with Doppler spectral bandwidth. The simulation results show that the Doppler shifts in slicks can decrease or increase, which depends on polarizations and incidence angles. The open ocean experiments carried out in 1997-1998 on the Black Sea performed at X- and Ka-bands with fixed incidence angle indicated that Doppler shifts in slicks can increase or decrease, which depends on radar wavelength and surface film characteristics [47, 48]. In addition, at wind speed 7-9 m/s, a strong reduction of Doppler shifts in slicks at X-band is observed in the wind-wave tank measurements performed at incidence angle of for $\mathrm{VV}$ polarization [4.5].

These qualitative results of Doppler shift and spectral bandwidth are potentially valuable for remote sensing of natural sea slicks floating on sea surfaces, although the present study is limited to L-band due to a severe computational burden. It needs to be pointed out that by developing GPU-based simulation codes the further investigation on this topic will be carried out at higher frequency bands such as X- and Ka-bands, which are more sensitive to oil slicks. In addition, the Doppler spectra differences between oil films with different film elasticity and the discrimination between oil films and look-alikes deserve further investigation, which goes beyond the scope of this paper. 


\section{Acknowledgement}

This work was supported by the National Science Foundation for Distinguished Young Scholars of China (Grant No.61225002).

\section{References}

[1] Y. Hisaki. Nonlinear inversion of the integral equation to estimate ocean wave spectra from hf radar. Radio Science, 1996.

[2] L. Wyatt. The measurement of the ocean wave directional spectrum from hf radar Doppler spectra. Radio Science, 1986.

[3] A. A. Mouche, F. Collard, B. Chapron, K.-F. Dagestad, G. Guitton, J. A. Johannessen, V. Kerbaol, and M. W. Hansen. On the use of Doppler shift for sea surface wind retrieval from SAR. IEEE Transactions on Geosciences and Remote Sensing, 2012.

[4] F. Saïd and H. Johnsen. Ocean surface wind retrieval from dual-polarized SAR data using the polarization residual Doppler frequency. IEEE Transactions on Geosciences and Remote Sensing, 2014.

[5] P. A. Hwang, M. A. Sletten, and J. V. Toporkov. A note on Doppler processing of coherent radar backscatter from the water surface: With application to ocean surface wave measurements. Journal of Geophysical Research, 2010.

[6] J. T. Johnson, R. J. Burkholder, J. V. Toporkov, D. R. Lyzenga, and W. J. Plant. A numerical study of the retrieval of sea surface height profiles from low grazing angle radar data. IEEE Transactions on Geosciences and Remote Sensing, 2009.

[7] N. Brau, F. Ziemer, A. Bezuglov, M. Cysewski, and G. Schymura. Seasurface current features observed by doppler radar. IEEE Transactions on Geosciences and Remote Sensing, 2008. 
[8] J. A. McGregor, E. M. Poulter, and M. J. Smith. Ocean surface currents obtained from microwave sea-echo Doppler spectra. Journal of Geophysical Research, 1997.

[9] Q. Huang, T. L. Dong, B. Y. Chen, Q. X. Li, and P. Chen. Vector scattering from one dimensional periodic perfectly conducting surface: $\mathrm{Te}$ polarization. Journal of Quantitative Spectroscopy and Radiative Transfer, 2014.

[10] H. J. Luo, G. D. Yang, Y. H. Wang, J. C. Shi, and Y. Du. Numerical studies of sea surface scattering with the GMRES-RP method. IEEE Transactions on Geosciences and Remote Sensing, 2014.

[11] A. G. Voronovich and V. U. Zavorotny. Full-polarization modeling of monostatic and bistatic radar scattering from a rough sea surface. IEEE Transactions on Antennas and Propagation, 2014.

[12] D. E. Barrick and J. B. Snider. The statistics of hf sea-echo doppler spectra. IEEE Transactions on Antennas and Propagation, 1977.

[13] D. B. Creamer, F. Henyey, R. Schult, and J. Wright. Improved linear representation of ocean surface waves. Journal of Fluid Mechanics, 1989.

[14] G. Soriano, M. Joelson, and M. Saillard. Doppler spectra from a twodimensional ocean surface at l-band. IEEE Transactions on Geosciences and Remote Sensing, 2006.

[15] F. Nouguier, C.-A. Guérin, and B. Chapron. "choppy wave" model for nonlinear gravity waves. Journal of Geophysical Research, 2009.

[16] B. J. West, K. A. Brueckner, R. S. Janda, D. M. Milder, and R. L. Milton. A new numerical method for surface hydrodynamics. Journal of Geophysical Research, 1987.

[17] R. Tao, Y. Li, X. Bai, and A. Waheed. Fractional weierstrass model for rough ocean surface and analytical derivation of its scattered field in a closed form. IEEE Transactions on Geosciences and Remote Sensing, 2012. 
[18] G. Lindgren and S. berg. First order stochastic lagrange model for asymmetric ocean waves. Journal of Offshore Mechanics and Arctic Engineering, 2009.

[19] J. T. Johnson, J. V. Toporkov, and G. S. Brown. A numerical study of backscattering from time-evolving sea surfaces: Comparison of hydrodynamic models. IEEE Transactions on Geosciences and Remote Sensing, 2001.

[20] D. Miret, G. Soriano, F. Nouguier, P. Forget, M. Saillard, and C.-A. Guérin. Sea surface microwave scattering at extreme grazing angle: Numerical investigation of the Doppler shift. IEEE Transactions on Geosciences and Remote Sensing, 2014.

[21] F. Nouguier, C.-A. Guérin, and G. Soriano. Analytical techniques for the Doppler signature of sea surfaces in the microwave regime-ii: nonlinear surfaces. IEEE Transactions on Geosciences and Remote Sensing, 2011.

[22] C. L. Rino, T. L. Crystal, A. K. Koide, H. D. Ngo, and H. Guthart. Numerical simulation of backscatter from linear and nonlinear ocean surface realizations. Radio Science, 1991.

[23] J. V. Toporkov and G. S. Brown. Numerical simulations of scattering from time-varying, randomly rough surfaces. IEEE Transactions on Geosciences and Remote Sensing, 2000.

[24] Y. H. Wang, Y. M. Zhang, M. X. He, and C. F. Zhao. Doppler spectra of microwave scattering fields from nonlinear oceanic surface at moderateand low-grazing angles. IEEE Transactions on Geosciences and Remote Sensing, 2012.

[25] F. Fois, P. Hoogeboom, F. L. Chevalier, and A. Stoffelen. An analytical model for the description of the full-polarimetric sea surface Doppler signature. Journal of Geophysical Research, 2015. 
[26] X. F. Li and X. J. Xu. Scattering and Doppler spectral analysis for twodimensional linear and nonlinear sea surfaces. IEEE Transactions on Geosciences and Remote Sensing, 2011.

[27] D. Nie, M. Zhang, N. Li, and W. Q. Jiang. Doppler spectra of electromagnetic fields scattered from two-dimensional fetch- and depth-limited nearshore sea surfaces. Journal of Quantitative Spectroscopy and Radiative Transfer, 2014.

[28] D. Nie, M. Zhang, C. Wang, and H. C. Yin. Study of microwave backscattering from two-dimensional nonlinear surfaces of finite-depth seas. IEEE Transactions on Geosciences and Remote Sensing, 2012.

[29] M. Migliaccio, F. Nunziata, and A. Gambardella. On the co-polarized phase difference for oil spill observation. International Journal of Remote Sensing, 2009.

[30] F. Nunziata, A. Gambardella, and M. Migliaccio. On the Mueller scattering matrix for SAR sea oil slick observation. IEEE Geoscience and Remote Sensing Letters, 2008.

[31] A. B. Salberg, O. Rudjord, and A. H. S. Solberg. Oil spill detection in hybrid-polarimetric SAR images. IEEE Transactions on Geosciences and Remote Sensing, 2014.

[32] A. H. S. Solberg. Remote sensing of ocean oil-spill pollution. Proceedings of the IEEE, 2012.

[33] A. H. S. Solberg, C. Brekke, and P. O. Husy. Oil spill detection in radarsat and envisat SAR images. IEEE Transactions on Geosciences and Remote Sensing, 2007.

[34] G. Franceschetti, A. Iodice, D. Riccio, G. Ruello, and R. Siviero. SAR raw signal simulation of oil slicks in ocean environments. IEEE Transactions on Geosciences and Remote Sensing, 2002. 
[35] F. Nunziata, P. Sobieski, and M. Migliaccio. The two-scale BPM scattering model for sea biogenic slicks contrast. IEEE Transactions on Geosciences and Remote Sensing, 2009.

[36] N. Pinel, C. Bourlier, and I. Sergievskaya. Two-dimensional radar backscattering modeling of oil slicks at sea based on the model of local balance: Validation of two asymptotic techniques for thick films. IEEE Transactions on Geosciences and Remote Sensing, 2014.

[37] N. Pinel, N. Déchamps, and C. Bourlier. Modeling of the bistatic electromagnetic scattering from sea surfaces covered in oil for microwave applications. IEEE Transactions on Geosciences and Remote Sensing, 2008.

[38] S. A. Ermakov, S. G. Salashin, and A. R. Panchenko. Film slicks on the sea surface and some mechanisms of their formation. Dynamics of Atmospheres and Oceans, 1992.

[39] A. Jenkins and S. Jacobs. Wave damping by a thin layer of viscous fluid. Physics of Fluids, 1997.

[40] P. P. Lombardini, B. Fiscella, P. Trivero, C. Cappa, and W. Garrett. Modulation of the spectra of short gravity waves by sea surface films: Slick detection and characterization with a microwave probe. J. Atmos. Ocean. Technol., 1989.

[41] T. Elfouhaily, B. Chapron, K. Katsaros, and D. Vandemark. A unified directional spectrum for long and short wind-driven waves. Journal of Geophysical Research, 1997.

[42] L. Tsang, J. A. Kong, K. H. Ding, and C. O. Ao. Scattering of Electromagnetic Waves: Numerical Simulations. Wiley-Interscience, New York, 2001.

[43] A. G. Voronovich and V. U. Zavorotny. Theoretical model for scattering of radar signals in ku- and c-bands from a rough sea surface with breaking waves. Waves in Random and Complex Media, 2001. 
[44] A. Stogryn. Equations for calculating the dielectric constant of saline water. IEEE Transactions on Microwave Theory and Techniques, 19(8):733-736, 1971.

[45] M. Gade, W. Alpers, S. A. Ermakov, H. Hhnerfuss, and P. A. Lange. Windwave tank measurements of bound and freely propagating short gravitycapillary waves. Journal of Geophysical Research, 1998.

[46] V. Karaev, M. Kanevsky, and E. Meshkov. The effect of sea surface slicks on the Doppler spectrum width of a backscattered microwave signal. Sensors, 2008.

[47] S. A. Ermakov, L. A. Sergievskaya, Y. B. Shchegolkov, S. V. Kijashko, J. C. Scott, and N. R. Stapleton. Anormal Doppler shifts of radar signals backscattered from marine slicks. In Proc. IEEE IGARSS, volume 5, pages 2986-2988.

[48] S. A. Ermakov, L. A. Sergievskaya, E. M. Zuikova, Y. B. Shchegolkov, J. C. Scott, and N. R. Stapleton. Field observations of radar backscatter modulation and radar Doppler shifts in slicks. In Proc. IEEE IGARSS, volume 4, pages 1513-1515. 